\title{
Milk and yogurt consumption are linked with higher bone mineral density but not with hip fracture: the Framingham Offspring Study
}

Shivani Sahni, PhD. ${ }^{1,2}$, Katherine L. Tucker, $\mathrm{PhD}^{3}$, Douglas P. Kiel, M.D., M.P.H. ${ }^{1,2}$, Ms. Lien Quach, M.P.H, M.S ${ }^{1}$, Ms. Virginia A. Casey, PhD ${ }^{1}$, and Dr. Marian T. Hannan, D.Sc., M.P.H. ${ }^{1,2}$ ${ }^{1}$ Institute for Aging Research, Hebrew SeniorLife, Boston MA

${ }^{2}$ Harvard Medical School, Boston, MA

${ }^{3}$ Northeastern University, Boston, MA

\section{Abstract}

Purpose-To examine associations of milk, yogurt, cheese, cream, most dairy (total dairy without cream) and fluid dairy (milk+yogurt) with bone density (BMD) at femoral neck (FN), trochanter (TR) and spine, and with incident hip fracture over 12-y follow-up in the Framingham Offspring Study.

Methods-3,212 participants completed a food frequency questionnaire (1991-1995 or 19951998) and were followed for hip fracture until 2007. 2,506 participants had DXA BMD (19962001). Linear regression was used to estimate adjusted mean BMD while Cox-proportional hazards regression was used to estimate adjusted hazard ratios (HR) for hip fracture risk. Final models simultaneously included dairy foods adjusting for each other.

Results-Mean baseline age was $55( \pm 1.6) y$, range: $26-85)$. Most dairy intake was positively associated with hip and spine BMD. Intake of fluid dairy and milk were related with hip but not spine BMD. Yogurt intake was associated with TR-BMD alone. Cheese and cream intakes were not associated with BMD. In final models, yogurt intake remained positively associated with TRBMD, while cream tended to be negatively associated with FN-BMD. Yogurt intake showed a weak protective trend for hip fracture [HR(95\%CI): $\leq 4$ serv/wk: $0.46(0.21-1.03)$ vs. >4 serv/wk: $0.43(0.06-3.27)]$. No other dairy groups showed a significant association (HRs range: $0.53-1.47$ ) with limited power ( $\mathrm{n}$, fractures $=43$ ).

Conclusion-Milk and yogurt intakes were associated with hip but not spine BMD, while cream may adversely influence BMD. Thus, not all dairy products are equally beneficial for the skeleton. Suggestive fracture results for milk and yogurt intakes need further confirmation.

Author for correspondence: Dr. Shivani Sahni, PhD, Phone: (617) 971-5382, Fax: (617) 971-5339, ShivaniSahni@ hsl.harvard.edu. Shivani Sahni, PhD., Institute for Aging Research, Hebrew SeniorLife and Harvard Medical School, 1200 Centre St., Boston, MA 02131

Katherine L. Tucker, PhD, Department of Health Sciences, Northeastern University School of Health Professionals, 316 Robinson Hall, Boston, MA 02115

Douglas P. Kiel, M.D., M.P.H., Institute for Aging Research, Hebrew SeniorLife and Harvard Medical School, 1200 Centre St., Boston, MA 02131

Ms. Lien Quach, M.P.H, M.S, Institute for Aging Research, Hebrew SeniorLife, 1200 Centre St., Boston, MA 02131

Ms. Virginia A. Casey, PhD, Institute for Aging Research, Hebrew SeniorLife, 1200 Centre St., Boston, MA 02131

Dr. Marian T. Hannan, D.Sc., M.P.H., Institute for Aging Research, Hebrew SeniorLife and Harvard Medical School, 1200 Centre St., Boston, MA 02131

Potential conflict of interest/disclosure: Dr. Sahni and Dr. Tucker have grants from General Mills Bell Institute of Health and Nutrition. 


\section{Keywords}

dairy; milk; yogurt; bone mineral density; hip fracture; dietary intake; bone health

\section{Introduction}

Osteoporosis is a major public health threat for an estimated 44 million Americans, or 55 percent of the people 50 years of age and older [1]. While many risk factors have been described, dietary factors represent an important and growing area of research for bone health. The 2010 dietary guidelines for Americans recommend consuming 3 cups per day of fat-free or low-fat milk and milk products for adults. Dairy foods are a complex source of essential nutrients, including protein, carbohydrates, fatty acids, calcium, phosphorus, potassium and magnesium, and are often fortified with vitamin D. It has been suggested that the health effects of dairy may be due to more than a single nutrient and, in fact, the effects of dairy foods may be greater than sum of their parts. A [2009] review by Heaney [2] and a report by the U.S. Dietary Guidelines advisory committee [3] both reported that 25 out of 32 observational studies and 11 out of 11 randomized controlled trials showed a significantly positive association between dairy food intake and bone mineral density (BMD) or bone mineral content (BMC). Milk and milk products may provide more sustained bone mass accrual than calcium supplements [2]. However, the nutrient profile changes with the type of dairy food. Choosing more dairy intake as fluid milk or yogurt rather than as cheese or cream can increase intake of potassium, vitamin A, and vitamin D and decrease intake of sodium, cholesterol, and saturated fatty acids. Therefore, it is important to examine the association of different types of dairy foods with bone health in adults and older adults.

\section{Methods}

Participants

In 1971, the Framingham Offspring Study was initiated by enrolling 5,124 adult children of the (original) Framingham Study and their spouses with the intent to identify risk factors in the etiology of coronary artery disease including familial factors [4]. Every 4-8 years, offspring participants have had physical examinations, blood chemistries, assessment of risk factors and questionnaires. Of the 5,124 originally enrolled Offspring cohort participants, 3,724 men and women completed a food frequency questionnaire (FFQ) in either 1991-95 or 1995-98 (baseline exam for this study) and were followed for hip fracture until 2007. Of these participants, 2506 men and women had a BMD assessment of hip and spine completed in 1996-2001 using dual-energy X-ray absorptiometry. We excluded participants with missing/incomplete FFQ (based on the criteria of $>12$ food items left blank in the FFQ) or with energy intakes $<2.51$ or $>16.74 \mathrm{MJ}(<600$ or $>4000 \mathrm{kcal} / \mathrm{d})$ at the baseline exam $(\mathrm{n}=59)$. We further excluded 68 participants due to missing covariate information on weight, height, physical activity index, menopause status or smoking status and another 13 participants who had a hip fracture prior to the baseline exam. Therefore, the final analytic sample included 3,212 Framingham Offspring study members for the hip fracture analyses and 2,733 members for the BMD analyses. All participants provided informed consent for their participation. This study was approved by the Institutional Review Board at the Institute for Aging Research, Hebrew SeniorLife.

\section{Assessment of dairy intake}

Usual dietary intake was assessed with the semi-quantitative, 126-item Willett FFQ at the baseline exam for this study (in either 1991-95 or 1995-98) [5-6]. This FFQ has been validated against multiple diet records and blood measures for many nutrients, including 
protein, in several populations [7-8]. An earlier version of this FFQ was validated against dietary records, with food intake for seven consecutive days four times during one-year interval, among 173 women from Nurses' Health Study [9]. The corrected correlation coefficients ranged from 0.57 to 0.94 for dairy products. Questionnaires were mailed to the study participants prior to their scheduled clinic visit. They were asked to complete the questionnaires, based on their intake over the previous year, and to bring them to the clinic examination, where they were reviewed with participants by clinic staff. Intake of dairy exposure variables was assessed in servings/week, using the food list section of the FFQ. Willett's questionnaire specifies the serve size for each dairy food as follows: - milk, skim/ low fat/whole ( $8 \mathrm{oz}$. glass), ice milk ( $1 / 2$ cup), cottage or ricotta cheese (1/2-cup), other cheese (1 slice or $1 \mathrm{oz}$. serving), cream (Tbs.), sour cream (Tbs.), ice cream (1/2-cup), cream cheese ( $1 \mathrm{oz}$.) and yogurt (1 cup). Milk intake was calculated as the sum of intake of skim milk, low fat milk, whole milk and ice milk. Cheese intake was calculated as the sum of intake of cottage/ricotta cheese, and other cheeses. Cream intake was calculated as the sum of intake of cream, sour cream, ice cream and cream cheese. Yogurt intake was also estimated in servings/week. A variable for most dairy intake was created, which included intake of milk, yogurt and cheese in servings/week. Furthermore, an additional variable for total dairy intake was created, which most dairy intake and cream groups.

\section{Bone Mineral Density}

BMD $\left(\mathrm{g} / \mathrm{cm}^{2}\right)$ of the hip (femoral neck and trochanter) and lumbar spine (L2-L4) was obtained. The right hip was scanned unless there was a history of fracture or hip replacement, in which case the left hip was scanned. BMD measurements were obtained in 1996-2001 using a dual-energy X-ray absorptiometry (DPX-L; GE Lunar Corporation). The $\mathrm{CV}$ for repeat scans over time using the DPX-L was 1.7 and $0.9 \%$ for the femoral neck and lumbar spine, respectively [10].

\section{Assessment of fracture}

Using the assessment protocol that has been reported previously [11], hip fractures were reported by hospitalization review, death review, interview at each 4-year examination or by telephone interview for participants unable to attend examinations. All but 3 reported hip fractures were confirmed by review of medical records and radiographic and operative reports. An incident hip fracture was defined as a first-time fracture of the proximal femur. Study participants were followed for hip fracture from the date of the dietary assessment through December, 2005.

\section{Potential confounding factors}

Sex, age (years), height (m), weight (kg), smoking (current vs. non-current), physical activity, and menopause status (yes/no) and current estrogen use (yes/no) in women, total energy (MJ/d), alcohol (g/d), caffeine (g/d), calcium supplement use (yes/no) and vitamin D supplement use (yes/no) were measured at the baseline exam for this study (in either 199195 or 1995-98). The dietary intakes and supplement use were assessed using the FFQ. Furthermore, intake of total energy and intake of dairy-specific nutrients such as dietary calcium, vitamin D, protein, magnesium, and phosphorus were measured using the food list section of the FFQ.

Height was measured without shoes, in inches, and weight was recorded in pounds with a standardized balance-beam scale. Smoking status, assessed by questionnaire, was used to classify individuals as either current smokers or former/never smokers. Physical activity was measured with the use of the Physical Activity Scale in the Elderly (PASE). Physical activity at the 1989-1992 exam was used for the participants who were missing physical activity assessment at the 1995-1998 exam. For those who remained missing, we used an 
average of physical activity from the 1983-1986 exam and the 1998-2001 exam. For estrogen use, women were divided into 2 groups: those currently using at the time of the exam compared with those who had never used or who had formerly used estrogen. Furthermore, menopause status (yes/no) and osteoporosis medication use (yes/no) were also determined at the same time as BMD measurement.

\section{Statistical analysis}

Analyses were conducted with both men and women combined. Interaction of dairy intake variables with sex was tested statistically. If the interaction term was significant at the level of $\mathrm{P}<0.05$ only then sex-specific analyses were conducted. All dairy intake exposures except yogurt, cheese and fluid dairy intake were normally distributed. Categorical variables were created for yogurt (no intake, medium intake: $\$ 4$ servings/week and high intake: $>4$ servings/week) and cheese intake (low intake: <2 servings/week, medium intake: $2-4$ servings/week and high intake: $>4$ servings/week). While tertile categories were created for fluid dairy intake. All dairy exposures were adjusted for total energy intake using the residual method [6]. Dairy intakes were regressed on total energy intake to create residuals, which were then added to the predicted dairy intake for the mean energy intake of the study population.

For BMD analyses, individual dairy intakes (in servings/week) were modeled as both continuous variables and as quartiles or appropriate categories. To determine the association between dairy intake variables and BMD at each site, we used multivariable linear regression to calculate regression coefficients $(\beta)$ estimating the difference in BMD associated with a 1-unit increase in dairy intake i.e. an increase of one serving per week. We used analysis of covariance to compare least-squares adjusted BMD among quartiles or categories of dairy intake, and tested for linear trend across quartiles.

For fracture analyses, individual dairy intakes were modeled as both continuous variables and as tertiles or appropriate categories. Tertiles were used for fracture, as there were insufficient numbers of hip fractures per quartile for comparison. We used Cox proportional hazards regression to calculate the hazard ratio (HR) and $95 \%$ confidence interval (CI) estimating the relative increase in risk of hip fracture for each 1 unit increase in each dairy intake variable. In addition, we compared the upper 2 tertiles or categories of dairy intake against the lowest tertile or category. We also tested for linear trend across tertiles or categories. Crude incidence rates in each tertile or category of the dairy exposure were also calculated.

The models were initially adjusted for sex, total energy intake, age, height and weight. Subsequent models were further adjusted for current cigarette smoking, calcium supplement use, vitamin D supplement use and, in women, menopause status and estrogen use. Physical activity, alcohol and caffeine were added to the full model, but these variables did not change the beta-coefficient by more than $10 \%$, and were not included in the final models. For analyses on the combined sample of men and women, we created an indicator variable to adjust for sex and menopause status (yes/no) simultaneously (group 1: men, group 2: pre menopausal women, group 3: post menopausal women).

All analyses were performed using SAS statistical software (SAS Institute Inc. SAS user's guide, version 9.1 Cary, NC: SAS Institute Inc, 2001). A nominal two-sided $P$-value of $<0.05$ was considered statistically significant for all of the analyses. 


\section{Results}

The mean age was 55y (SD: 1.6, range: 26-85) at the baseline exam (Table 1). Ninety two percent of the participants did not reach the recommendation of 3 servings of dairy per day. Furthermore, only $24 \%$ of participants reached the calcium RDA for their age and gendergroup, while $12 \%$ of the participants reached the vitamin D RDA for their age group. Fortythree hip fractures occurred over the 12-year follow-up period. Participants in the highest quartile of total dairy intake had relatively high intakes of dairy specific nutrients- protein, calcium, vitamin D, magnesium and phosphorus compared to participants in the lowest quartile of total dairy intake (Table 2). In contrast, participants in the highest quartile of cream intake had lower intakes of dairy specific nutrients compared to the participants in the lowest quartile of cream intake.

\section{Association with BMD}

The association of dairy intake with BMD was similar in men and women and the $P$ values for the statistical interaction of dairy intake variables with gender ranged from $P=0.10-0.98$. Therefore, mean differences in BMD compared to the reference quartile (Q1) or category of dairy intake are presented in the combined sample of men and women (Table 3). Participants in the highest quartile of milk intake showed a positive but non-significant trend with femoral neck BMD ( $P$ value: Q4 vs. Q1: $0.23, \mathrm{Q} 3$ vs. Q1: $0.52, \mathrm{P}$ trend: 0.08$)$ and significant positive association with trochanter BMD $(P$ value: Q4 vs. Q1=0.23, Q3 vs. $\mathrm{Q} 1=0.09, P$ trend=0.05) compared to participants in the lowest quartile of milk intake. No significant associations were observed for milk intake and BMD at the lumbar spine ( $P$ trend=0.29). Participants with high yogurt intake ( $>4$ servings/week) had higher BMD at the trochanter compared to those with no intake ( $P$ value for high yogurt intake vs. no intake $=$ 0.03 , Table 3 ), while no significant associations were observed for other bone sites ( $P$ range: $0.27-0.32$ ). Similarly, no significant associations were observed for cheese intake ( $P$ trend range: $0.29-0.54)$ or cream intake ( $P$ trend range: $0.39-0.42$ ). Participants in the highest quartile (quartile 3-4) of most dairy (excluding the cream group) had significantly higher BMD at the hip (FN-BMD: $P$ value for Q4 vs. Q1 $=0.008, \mathrm{Q} 3$ vs. Q1 $=0.03, P$ trend: 0.001 and for TR-BMD: $P$ value for Q4 vs. Q1=0.004, Q3 vs. Q1=0.06, $P$ trend: 0.02 ) and spine (LS-BMD: $P$ value for Q4 vs. Q1=0.02, Q3 vs. Q1=0.06, $P$ trend: 0.02 ) compared to participants in the lowest quartile of intake. Participants in the highest quartile of fluid dairy (milk and yogurt intake) had significantly higher BMD at the hip (FN-BMD: $P$ value for Q4 vs. $\mathrm{Q} 1=0.03, \mathrm{Q} 4$ vs. $\mathrm{Q} 2=0.006)$ compared to the participants in the lowest two quartiles (Q1-Q2). The same was also true for TR-BMD ( $P$ trend: 0.02$)$ while no significant associations were observed for LS-BMD ( $P$ trend: 0.15 ).

When intake of milk, yogurt, cheese and cream were simultaneously included in the model, yogurt intake remained positively associated with trochantric BMD, while cream intake tended to be negatively associated with femoral neck BMD (Table 4). Milk and yogurt intake showed a positive but non-significant trend with femoral neck BMD $(P=0.06$ and 0.09 respectively). No associations were observed for cheese intake or for lumbar spine BMD.

\section{Association with hip fracture}

Participants in the higher tertiles (T2-3) of milk intake had a lower but non-significant risk of hip fracture than those in the lowest tertile (T1) (HRs: T2: 0.78; 95\%CI: 0.37-1.63, T3: 0.50 ; 95\%CI: $0.22-1.13, P$ trend=0.09). Similarly, participants in the higher categories $(\mathrm{C} 2-$ 3 ) of yogurt intake showed a protective but non-significant association with the risk of hip fracture as compared to participants in the lowest category (C1) (HRs: C2: 0.39; 95\%CI: 0.15-1.02, C3: 0.57; 95\%CI: 0.19-1.68, $P$ trend=0.10). Participants in the highest tertile (T3) of fluid dairy intake had a lower risk of hip fracture than those in the lowest tertile (T1, 
$P$ for T3 vs. T1: 0.05 ) (HRs: T2: 0.92; 95\%CI: 0.46-1.87, T3: 0.40; 95\%CI: $0.17-0.99, P$ trend $=0.06)$. None of the other dairy groups showed a significant association $(P$ trend range: $0.25-0.61$, data not shown).

\section{Discussion}

In this group of largely middle-aged men and women, total dairy intakes were significantly associated with higher BMD at the hip and spine. Milk and yogurt showed positive associations with trochantric BMD while cream intake tended to be adversely associated with femoral neck BMD. Results for fracture were not significant, but there were weak protective trends for milk and yogurt intake. Previous studies have shown that individual nutrients usually found in dairy products, such as calcium and vitamin D [12], protein [1314] and potassium [15] are beneficial for bone health, while high intakes of sodium [16] may have a negative impact. Studies have also explored the role of synergy between these nutrients. Few studies have directly compared different types of dairy foods even though it has been suggested that dairy foods are not equivalent vehicles of calcium due to their different protein, sodium, potassium and vitamin A contents [17]. Furthermore, it should be noted that the absorption of calcium from dairy foods is further dependent on vitamin $\mathrm{D}$ intake levels. The total vitamin D intake (mean: $309 \mathrm{IU} / \mathrm{d}$ ) in this cohort was lower than the RDA for vitamin D intake. Based on the nutrient profiles of dairy foods, it can be predicted that milk [has the largest calcium to sodium ratio (2.4)] and yogurt may be advantageous to bone; whereas certain cheeses, which have high sodium and polyphosphate, may be less advantageous [17]. Due to its low nutrient density, cream, may actually be disadvantageous to bone health (Table 2). Furthermore, calcium from yogurt has higher bioavailability than from milk due to the acidic $\mathrm{pH}$ of the yogurt, which ionizes the calcium, facilitating absorption [18]. Whereas the manufacture of fresh, aged, and processed cheeses results in products that differ markedly in their content of dairy specific nutrients compared to milk [17]. Therefore, the association between cheese and BMD needs further exploration with detailed data on the type of cheese. Milk remains the most studied dairy food; far less is known about the associations of other dairy foods such as yogurt, cheese and cream with bone health.

\section{Evidence from observational studies}

Several cross-sectional studies have reported a positive link between childhood milk consumption and bone density later in life [19-22]. Evidence for a beneficial role of milk intake on fracture is less convincing [23-24,19]. A cross-sectional study of women (aged 44-74 years) found consistent positive associations between self-reported milk consumption before the age of 25 years and BMD at total hip, femoral neck, trochanter, intertrochanter $(P<0.05$ each), and Ward's triangle $(P<0.005)$ [22]. Results from 581 white elderly women (mean age: 71 years) in the Rancho Bernardo Study showed that regular milk consumption in youth was associated with better BMD at cortical and trabecular sites [25]. Similarly, women aged 20-49 years from the third National Health and Nutrition Examination Survey, who consumed $<1$ serving of milk per week during childhood (ages 5-12 years) were found to have 5.6\% less bone mineral content (BMC), and 3\% lower hip BMC and BMD $(P<0.02)$ than those who consumed $>1$ serving per day [19]. In the same study, women aged 50 years or older with low milk intake during childhood were found to have twice the risk of lifetime fracture [OR: 2.02 (95\% CI: 1.13, 3.59), $P<0.05$ ] [19]. On the other hand, a 12 year prospective study of 77,761 women (aged 34-59 years) from the Nurses' Health Study found no evidence that higher adult milk intake ( $\geq 2$ glasses of milk/day versus $\leq$ glass/ week) reduced incidence of hip fracture [RR: 1.45 (95\% CI: 0.87-2.43)] or forearm fracture [RR: 1.05 (95\% CI: 0.88-1,25)] [24]. An 18-year prospective analysis in 72,337 postmenopausal women from the same study also showed no association between milk 
intake and hip fracture risk [26]. Furthermore, a meta-analysis of milk consumption and hip fracture found no overall association [Pooled RR for each additional glass of milk/day for men: 0.91 (95\% CI: 0.8-1.01) and for women: 0.99 (95\% CI: 0.96-1.02), Qtest $P=0.37$ ] [23].

\section{Evidence from randomized controlled trials}

Randomized controlled trials that primarily focused on change in BMD over time as the outcome demonstrated a protective effect of dairy intake against bone loss. When women aged 18-30 years were randomized to either a control group ( $<800 \mathrm{mg} / \mathrm{d}$ dietary calcium), a medium dairy intake group (1000-1100 mg/d dietary calcium), or a high dairy intake group (1200-1300 mg/d dietary calcium), higher dairy intakes positively impacted percent change in total hip BMD $(P=0.006)$ and total hip BMC $(P=0.02)$ over 1-year. The average bone loss in the control group was $0.45 \%$ compared with $0.43 \%$ gain in the two dairy groups combined $(P=0.008)$ [27]. A 3-year randomized study of 37 premenopausal women (aged 30 to 42 years) found that women who increased their dietary calcium through dairy products had significantly less change in vertebral bone density $(-0.4 \pm 0.9 \%)$ than age and weight matched controls $(-2.9 \pm 0.8 \%, \mathrm{p}<0.001)$ [28]. Similar results have been reported from other randomized controlled trials, including postmenopausal Chinese women in Malaysia [29], Greece [30] and South Australia [31]. In two trials, adult women randomly assigned to receive $40 \mathrm{mg} /$ day milk basic protein (MBP) for six months had improved left calcaneus BMD [32] and L2-L4 BMD than the placebo group [33]. A 2year randomized trial of fortified milk supplementation in older men (mean age $61.9 \pm 7.7$ years) showed that the net beneficial effect of milk supplementation persisted at femoral neck and ultradistal radius BMD (1.8\% and $1.5 \%$, respectively; $P=0.10)$ after 18 months, during which time no fortified milk was provided. Similarly, net beneficial effects at the total hip $(0.8 \% ; P=0.17)$ also persisted 18 months later $(0.7 \% ; P=0.10)$ [34]. Taken together the results of these randomized controlled trials are consistent with the results for total dairy intake with BMD in the current study.

Considered together, the evidence suggests a significant role for dairy intake and related nutrients in maintaining BMD. However, the evidence for hip fracture remains weak. Because hip fractures occur infrequently, the most practical study design is a prospective cohort study. Results from two of the largest studies to date on this topic have shown no association between dairy intake and the risk of hip fracture [23-24]; although a metaanalysis suggested that more data are required, particularly for men [23]. Compared to these studies, the current study included younger participants, with fewer fractures. Thus power was limited. Although we observed a protective trend for specific dairy groups, the results did not reach statistical significance. Still, we observed a marginally significant protective effect of milk and yogurt intake against hip fracture risk.

The current study is unique in that we used data from a population-based cohort of middleaged individuals followed for up to 12 years. Additionally, both BMD and fracture data were available for analyses. However, this study was limited by the small number of hip fractures that have occurred to-date. With the available sample size, we had $70 \%$ power to detect RR of 0.45 . Other limitations include the possibility of misspecification of dietary intake. We used a FFQ, which is semi-quantitative, and dietary data were available only at the baseline exam. Therefore, we were unable to adjust for possible changes in diet over follow-up. However, we examined not just total dairy intake but also the association of specific dairy food groups with both BMD and hip fracture risk. Dairy foods such as milk, yogurt and cream have dissimilar nutritional profiles that may play a role in the way they affect bone health. In any observational study, residual confounding may occur despite our attempts to control for several potential confounders. Lastly, the results of this study are generalizable primarily to non-Hispanic white men and women. 
In conclusion, our results suggest that, among this population of largely middle-aged men and women, higher total dairy, milk and yogurt intakes are associated with higher BMD. This effect was not observed for cheese and cream intake. No significant associations were observed for dairy intake and the risk of hip fracture except for milk and yogurt intake, which showed a marginally significant protective effect. Future studies should consider nutrient profiles of specific dairy groups while researching into their associations with bone health. Lastly, more data are needed to better understand the association of dairy foods with hip fracture risk in men and women.

\section{Acknowledgments}

This work was supported by the National Institute of Arthritis and Musculoskeletal and Skin Diseases (AR \# 053205 and also AR/AG41398) and by the National Heart Lung and Blood Institute's Framingham Heart Study (N01-HC-25195), the Melvin First Young Investigator Award and General Mills Bell Institute of Health and Nutrition.

Authors would like to thank Miss. Brianne Jeffrey from the Institute for Aging Research, Hebrew SeniorLife for her general assistance with this project.

\section{References}

1. National Osteoporosis Foundation. [Accessed September 5, 2012] Fast Facts. http://www.nof.org/ node $/ 40$

2. Heaney RP. Dairy and bone health. J Am Coll Nutr. 2009; 28(Suppl 1):82S-90S. 28/ Supplement_1/82S [pii]. [PubMed: 19571166]

3. Dietary guidelines for Americans. Dietary Guidelines Advisory Committee Report. 6. U.S. Department of Health and Human Services and U.S. Department of Agriculture, U.S. Government; Washington DC: 2005.

4. Kannel WB, Feinleib M, McNamara PM, Garrison RJ, Castelli WP. An investigation of coronary heart disease in families. The Framingham offspring study. Am J Epidemiol. 1979; 110 (3):281290. [PubMed: 474565]

5. Rimm EB, Giovannucci EL, Stampfer MJ, Colditz GA, Litin LB, Willett WC. Reproducibility and validity of an expanded self-administered semiquantitative food frequency questionnaire among male health professionals. Am J Epidemiol. 1992; 135(10):1114-1126. discussion 1127-1136. [PubMed: 1632423]

6. Willett W, Stampfer MJ. Total energy intake: implications for epidemiologic analyses. Am J Epidemiol. 1986; 124 (1):17-27. [PubMed: 3521261]

7. Ascherio A, Stampfer MJ, Colditz GA, Rimm EB, Litin L, Willett WC. Correlations of vitamin A and $\mathrm{E}$ intakes with the plasma concentrations of carotenoids and tocopherols among American men and women. J Nutr. 1992; 122 (9):1792-1801. [PubMed: 1512628]

8. Jacques PF, Sulsky SI, Sadowski JA, Phillips JC, Rush D, Willett WC. Comparison of micronutrient intake measured by a dietary questionnaire and biochemical indicators of micronutrient status. Am J Clin Nutr. 1993; 57 (2):182-189. [PubMed: 8424386]

9. Salvini S, Hunter DJ, Sampson L, Stampfer MJ, Colditz GA, Rosner B, Willett WC. Food-based validation of a dietary questionnaire: the effects of week-to-week variation in food consumption. Int J Epidemiol. 1989; 18 (4):858-867. [PubMed: 2621022]

10. Kiel DP, Mercier CA, Dawson-Hughes B, Cali C, Hannan MT, Anderson JJ. The effects of analytic software and scan analysis technique on the comparison of dual X-ray absorptiometry with dual photon absorptiometry of the hip in the elderly. J Bone Miner Res. 1995; 10(7):11301136.10.1002/jbmr.5650100719 [PubMed: 7484290]

11. Kiel DP, Felson DT, Anderson JJ, Wilson PW, Moskowitz MA. Hip fracture and the use of estrogens in postmenopausal women. The Framingham Study. N Engl J Med. 1987; 317 (19): 1169-1174. [PubMed: 3657888] 
12. Dawson-Hughes B, Harris SS, Krall EA, Dallal GE. Effect of calcium and vitamin D supplementation on bone density in men and women 65 years of age or older. N Engl J Med. 1997; 337(10):670-676.10.1056/NEJM199709043371003 [PubMed: 9278463]

13. Hannan MT, Tucker KL, Dawson-Hughes B, Cupples LA, Felson DT, Kiel DP. Effect of dietary protein on bone loss in elderly men and women: the Framingham Osteoporosis Study. J Bone Miner Res. 2000; 15(12):2504-2512.10.1359/jbmr.2000.15.12.2504 [PubMed: 11127216]

14. Sahni S, Cupples LA, McLean RR, Tucker KL, Broe KE, Kiel DP, Hannan MT. Protective effect of high protein and calcium intake on the risk of hip fracture in the Framingham offspring cohort. J Bone Miner Res. 2010; 25(12):2770-2776.10.1002/jbmr.194 [PubMed: 20662074]

15. Tucker KL, Hannan MT, Chen H, Cupples LA, Wilson PW, Kiel DP. Potassium, magnesium, and fruit and vegetable intakes are associated with greater bone mineral density in elderly men and women. Am J Clin Nutr. 1999; 69 (4):727-736. [PubMed: 10197575]

16. Devine A, Criddle RA, Dick IM, Kerr DA, Prince RL. A longitudinal study of the effect of sodium and calcium intakes on regional bone density in postmenopausal women. Am J Clin Nutr. 1995; 62 (4):740-745. [PubMed: 7572702]

17. Weinsier RL, Krumdieck CL. Dairy foods and bone health: examination of the evidence. Am J Clin Nutr. 2000; 72 (3):681-689. [PubMed: 10966884]

18. Unal G, El SN, Kilic S. In vitro determination of calcium bioavailability of milk, dairy products and infant formulas. Int J Food Sci Nutr. 2005; 56(1):13-22. RR54364580742841 [pii]. 10.1080/09637480500081423 [PubMed: 16019311]

19. Kalkwarf HJ, Khoury JC, Lanphear BP. Milk intake during childhood and adolescence, adult bone density, and osteoporotic fractures in US women. Am J Clin Nutr. 2003; 77 (1):257-265. [PubMed: 12499350]

20. Kull M, Kallikorm R, Lember M. Impact of molecularly defined hypolactasia, self-perceived milk intolerance and milk consumption on bone mineral density in a population sample in Northern Europe. Scand J Gastroenterol. 2009; 44(4):415-421. 906066060 [pii]. 10.1080/00365520802588117 [PubMed: 19039706]

21. McCabe LD, Martin BR, McCabe GP, Johnston CC, Weaver CM, Peacock M. Dairy intakes affect bone density in the elderly. Am J Clin Nutr. 2004; 80(4):1066-1074. 80/4/1066 [pii]. [PubMed: 15447921]

22. Murphy S, Khaw KT, May H, Compston JE. Milk consumption and bone mineral density in middle aged and elderly women. BMJ. 1994; 308 (6934):939-941. [PubMed: 8173399]

23. Bischoff-Ferrari HA, Dawson-Hughes B, Baron JA, Kanis JA, Orav EJ, Staehelin HB, Kiel DP, Burckhardt P, Henschkowski J, Spiegelman D, Li R, Wong JB, Feskanich D, Willett WC. Milk intake and risk of hip fracture in men and women: a meta-analysis of prospective cohort studies. $\mathrm{J}$ Bone Miner Res. 2011; 26(4):833-839.10.1002/jbmr.279 [PubMed: 20949604]

24. Feskanich D, Willett WC, Stampfer MJ, Colditz GA. Milk, dietary calcium, and bone fractures in women: a 12-year prospective study. Am J Public Health. 1997; 87 (6):992-997. [PubMed: 9224182]

25. Soroko S, Holbrook TL, Edelstein S, Barrett-Connor E. Lifetime milk consumption and bone mineral density in older women. Am J Public Health. 1994; 84 (8):1319-1322. [PubMed: 8059895]

26. Feskanich D, Willett WC, Colditz GA. Calcium, vitamin D, milk consumption, and hip fractures: a prospective study among postmenopausal women. Am J Clin Nutr. 2003; 77 (2):504-511. [PubMed: 12540414]

27. Teegarden D, Legowski P, Gunther CW, McCabe GP, Peacock M, Lyle RM. Dietary calcium intake protects women consuming oral contraceptives from spine and hip bone loss. J Clin Endocrinol Metab. 2005; 90(9):5127-5133. jc.2004-0924 [pii]. 10.1210/jc.2004-0924 [PubMed: 15998785]

28. Baran D, Sorensen A, Grimes J, Lew R, Karellas A, Johnson B, Roche J. Dietary modification with dairy products for preventing vertebral bone loss in premenopausal women: a three-year prospective study. J Clin Endocrinol Metab. 1990; 70 (1):264-270. [PubMed: 2294135] 
29. Chee WS, Suriah AR, Chan SP, Zaitun Y, Chan YM. The effect of milk supplementation on bone mineral density in postmenopausal Chinese women in Malaysia. Osteoporos Int. 2003; 14(10): 828-834.10.1007/s00198-003-1448-6 [PubMed: 12915959]

30. Moschonis G, Manios Y. Skeletal site-dependent response of bone mineral density and quantitative ultrasound parameters following a 12-month dietary intervention using dairy products fortified with calcium and vitamin D: the Postmenopausal Health Study. Br J Nutr. 2006; 96(6):1140-1148. S0007114507244423 [pii]. [PubMed: 17181890]

31. Cleghorn DB, O'Loughlin PD, Schroeder BJ, Nordin BE. An open, crossover trial of calciumfortified milk in prevention of early postmenopausal bone loss. Med J Aust. 2001; 175 (5):242245. [PubMed: 11587253]

32. Aoe S, Toba Y, Yamamura J, Kawakami H, Yahiro M, Kumegawa M, Itabashi A, Takada Y. Controlled trial of the effects of milk basic protein (MBP) supplementation on bone metabolism in healthy adult women. Biosci Biotechnol Biochem. 2001; 65 (4):913-918. [PubMed: 11388472]

33. Aoe S, Koyama T, Toba Y, Itabashi A, Takada Y. A controlled trial of the effect of milk basic protein (MBP) supplementation on bone metabolism in healthy menopausal women. Osteoporos Int. 2005; 16(12):2123-2128.10.1007/s00198-005-2012-3 [PubMed: 16133638]

34. Daly RM, Petrass N, Bass S, Nowson CA. The skeletal benefits of calcium- and vitamin D3fortified milk are sustained in older men after withdrawal of supplementation: an 18-mo follow-up study. Am J Clin Nutr. 2008; 87(3):771-777. 87/3/771 [pii]. [PubMed: 18326617] 
Table 1

Characteristics of men and women in the Framingham Offspring Cohort.

\begin{tabular}{|c|c|}
\hline Characteristics & $($ Mean \pm SD) or $(\%)$ \\
\hline Women $(\mathrm{n}, \%)$ & $1681(55.8 \%)$ \\
\hline Age (years) & $54.9 \pm 9.6($ range $26-85)$ \\
\hline Weight (lb) & $171 \pm 37$ \\
\hline Height (cm) & $168 \pm 9$ \\
\hline Physical Activity (PASE) & $37 \pm 7$ \\
\hline Total energy intake (Kcal/d) & $1853 \pm 612$ \\
\hline Protein $(\mathrm{g} / \mathrm{d})$ & $77 \pm 27$ \\
\hline Total calcium (mg/d) & $829 \pm 436$ \\
\hline Calcium supplement users (\%) & 21.7 \\
\hline Total vitamin D (IU/d) & $309 \pm 248$ \\
\hline Vitamin D supplement users (\%) & 28.6 \\
\hline Magnesium (mg/d) & $302 \pm 110$ \\
\hline Phosphorus (mg/d) & $1213 \pm 438$ \\
\hline Total milk:(servings/week) & $5.8 \pm 6.2$ \\
\hline Total cheese:(servings/week) & $2.8 . \pm 3.2$ \\
\hline Total cream:(servings/week) & $4.2 \pm 7.2$ \\
\hline Yogurt:(servings/week) & $0.86 \pm 1.96$ \\
\hline Most dairy intake:(servings/week) & $9.5 \pm 7.5$ \\
\hline Fluid dairy (servings/week) & $6.7 \pm 6.6$ \\
\hline Current smokers (\%) & 17.0 \\
\hline FN-BMD $\left(\mathrm{g} / \mathrm{cm}^{2}\right)^{a}$ & $0.913 \pm 0.149$ \\
\hline $\operatorname{TR}-\mathrm{BMD}\left(\mathrm{g} / \mathrm{cm}^{2}\right)^{a}$ & $0.788 \pm 0.161$ \\
\hline $\mathrm{L} 2-4 \mathrm{BMD}\left(\mathrm{g} / \mathrm{cm}^{2}\right)^{a}$ & $1.226 \pm 0.219$ \\
\hline Hip fractures (n) $b$ & 43 \\
\hline$=2506$ for BMD analyses and & \\
\hline
\end{tabular}




\section{Table 2}

Distribution of selected nutrients within quartiles of total dairy intake and cream intake, in men and women in the Framingham Offspring Cohort.

\begin{tabular}{lcccc}
\hline Nutrients & \multicolumn{3}{c}{ Means \pm SD of nutrients by quartiles of total dairy intake (servings/week) } \\
\cline { 2 - 5 } & Quartile 1 (n=613) & Quartile 2 (n=591) & Quartile 3 (n=583) & Quartile 4 (n=607) \\
\hline Median intake (serv/week) & 5.50 & 7.94 & 12.44 & 23.44 \\
Protein & $78 \pm 27$ & $71 \pm 25^{*}$ & $75 \pm 25^{*}$ & $85 \pm 28^{*}$ \\
Calcium & $711 \pm 375$ & $692 \pm 342$ & $861 \pm 408^{*}$ & $1110 \pm 492^{*}$ \\
Vitamin D & $254 \pm 229$ & $277 \pm 246$ & $328 \pm 246^{*}$ & $391 \pm 265^{*}$ \\
Magnesium & $307 \pm 110$ & $277 \pm 101^{*}$ & $295 \pm 106^{*}$ & $335 \pm 115^{*}$ \\
Phosphorus & $1164 \pm 412$ & $1070 \pm 377^{*}$ & $1193 \pm 404$ & $1436 \pm 467^{*}$ \\
\hline & Means \pm SD of nutrients by quartiles of cream intake (servings/week) \\
\cline { 2 - 5 } & Quartile $1(\mathrm{n}=619)$ & Quartile 2(n=622) & Quartile 3(n=610) & Quartile 4 (n=598) \\
\hline Median intake (serv/week) & 0.47 & 0.94 & 1.94 & 7.47 \\
Protein & $91 \pm 27$ & $71 \pm 22^{*}$ & $71 \pm 26^{*}$ & $77 \pm 26^{*}$ \\
Calcium & $1025 \pm 485$ & $783 \pm 404^{*}$ & $752 \pm 410^{*}$ & $802 \pm 395^{*}$ \\
Vitamin D & $385 \pm 272$ & $308 \pm 250^{*}$ & $280 \pm 247^{*}$ & $275 \pm 216^{*}$ \\
Magnesium & $367 \pm 112$ & $282 \pm 94^{*}$ & $274 \pm 106^{*}$ & $293 \pm 102^{*}$ \\
Phosphorus & $1462 \pm 441$ & $1108 \pm 360^{*}$ & $1093 \pm 422^{*}$ & $1198 \pm 417^{*}$ \\
\hline
\end{tabular}

Means \pm SD significantly different from the mean in the lowest quartile of dairy intake, $P<0.05$ 
Table 3

Mean difference in BMD $\left(\mathrm{g} / \mathrm{cm}^{2}\right)$ by quartiles or categories of dairy intake (servings/week) in men and women in the Framingham Offspring Cohort.

\begin{tabular}{|c|c|c|c|c|}
\hline \multirow[t]{2}{*}{ Dairy intake (serv./week) in quartiles (Q1-Q4) or categories } & \multirow[t]{2}{*}{ Median Intake } & \multicolumn{3}{|c|}{ Mean $\pm \mathrm{SE}^{a}(\mathrm{n}=2,479)$} \\
\hline & & FN-BMD & TR-BMD & LS-BMD \\
\hline \multicolumn{5}{|l|}{ Milk } \\
\hline Q1 (Lowest) & 0.47 & $0.9136 \pm 0.005$ & $0.7853 \pm 0.005$ & $1.2234 \pm 0.008$ \\
\hline Q2 & 3.00 & $0.9044 \pm 0.005$ & $0.7790 \pm 0.005$ & $1.2149 \pm 0.008$ \\
\hline Q3 & 6.00 & $0.9179 \pm 0.005$ & $0.7966 \pm 0.005$ & $1.2349 \pm 0.008$ \\
\hline Q4 (Highest) & 12.50 & $0.9216 \pm 0.005$ & $0.7935 \pm 0.005$ & $1.2287 \pm 0.008$ \\
\hline Ptrend & & 0.08 & 0.05 & 0.29 \\
\hline \multicolumn{5}{|l|}{ Yogurt } \\
\hline No intake & 0 & $0.9142 \pm 0.003$ & $0.7870 \pm 0.003$ & $1.2246 \pm 0.005$ \\
\hline Medium intake ( $\leqslant$ serv./wk) & 0.47 & $0.9133 \pm 0.004$ & $0.7921 \pm 0.004$ & $1.2305 \pm 0.006$ \\
\hline High intake (>4 serv./wk) & 7.00 & $0.9327 \pm 0.009$ & $0.8089 \pm 0.009$ & $1.2415 \pm 0.016$ \\
\hline Ptrend & & 0.32 & 0.05 & 0.27 \\
\hline \multicolumn{5}{|l|}{ Cheese } \\
\hline Low intake (<2 serv./wk) & 0.94 & $0.9138 \pm 0.004$ & $0.7901 \pm 0.004$ & $1.2257 \pm 0.006$ \\
\hline Medium intake (2-4 serv./wk) & 3.00 & $0.9150 \pm 0.004$ & $0.7869 \pm 0.004$ & $1.2253 \pm 0.007$ \\
\hline High intake (>4 serv./wk) & 6.00 & $0.9184 \pm 0.006$ & $0.7977 \pm 0.006$ & $1.2402 \pm 0.009$ \\
\hline P trend & & 0.54 & 0.48 & 0.29 \\
\hline \multirow[t]{2}{*}{ Dairy intake (serv./week)in quartiles (Q1-Q4) or categories } & Median Intake & \multicolumn{3}{|c|}{ Mean $\pm \mathrm{SE}^{a}(\mathrm{n}=2506)$} \\
\hline & & FN-BMD & TR-BMD & LS-BMD \\
\hline \multicolumn{5}{|l|}{ Cream } \\
\hline Q1 (Lowest) & 0.47 & $0.9130 \pm 0.005$ & $0.7850 \pm 0.005$ & $1.2282 \pm 0.008$ \\
\hline Q2 & 0.94 & $0.9180 \pm 0.005$ & $0.7971 \pm 0.005$ & $1.2286 \pm 0.008$ \\
\hline Q3 & 1.94 & $0.9121 \pm 0.005$ & $0.7816 \pm 0.005$ & $1.2223 \pm 0.008$ \\
\hline Q4 (Highest) & 7.47 & $0.9090 \pm 0.005$ & $0.7867 \pm 0.005$ & $1.2211 \pm 0.008$ \\
\hline P trend & $<.0001$ & 0.39 & 0.59 & 0.42 \\
\hline \multicolumn{5}{|l|}{ Most Dairy (w/o cream group) } \\
\hline Q1 (Lowest) & 3.47 & $0.9064 \pm 0.005$ & $0.7793 \pm 0.005$ & $1.2105 \pm 0.008$ \\
\hline Q2 & 5.47 & $0.9048 \pm 0.005$ & $0.7813 \pm 0.005$ & $1.2251 \pm 0.008$ \\
\hline Q3 & 8.97 & $0.9211 \pm 0.005$ & $0.7924 \pm 0.005$ & $1.2316 \pm 0.008$ \\
\hline Q4 (Highest) & 18.50 & $0.9247 \pm 0.005$ & $0.7987 \pm 0.005$ & $1.2354 \pm 0.008$ \\
\hline P trend & $<.0001$ & 0.001 & 0.02 & 0.02 \\
\hline \multicolumn{5}{|l|}{ Milk+yogurt } \\
\hline Q1 (Lowest) & 0.47 & $0.9097 \pm 0.005$ & $0.7864 \pm 0.005$ & $1.2254 \pm 0.008$ \\
\hline Q2 & 3.00 & $0.9054 \pm 0.005$ & $0.7797 \pm 0.005$ & $1.2175 \pm 0.008$ \\
\hline Q3 & 7.00 & $0.9205 \pm 0.005$ & $0.7974 \pm 0.005$ & $1.1230 \pm 0.008$ \\
\hline Q4 (Highest) & 17.50 & $0.9240 \pm 0.005$ & $0.7973 \pm 0.005$ & $1.2371 \pm 0.008$ \\
\hline P trend & $<.0001$ & 0.006 & 0.02 & 0.15 \\
\hline
\end{tabular}


${ }^{a} \mathrm{Mean} \pm \mathrm{SE}$ represent the mean difference in BMD compared to the reference quartile (Q1). Means are significantly different from means of Q1: * $P<0.05$,

${ }^{\dagger} P=0.1$

${ }^{a}$ Mean \pm SE represent the mean difference in BMD compared to the reference quartile (Q1), adjusting for age, sex, total energy intake, weight, height and menopause, estrogen, calcium supplement use, and vitamin D supplement use and current smoking.

Means are significantly different from means of Q1:

${ }^{*} P<0.05$,

${ }^{\dagger} P=0.1$ 


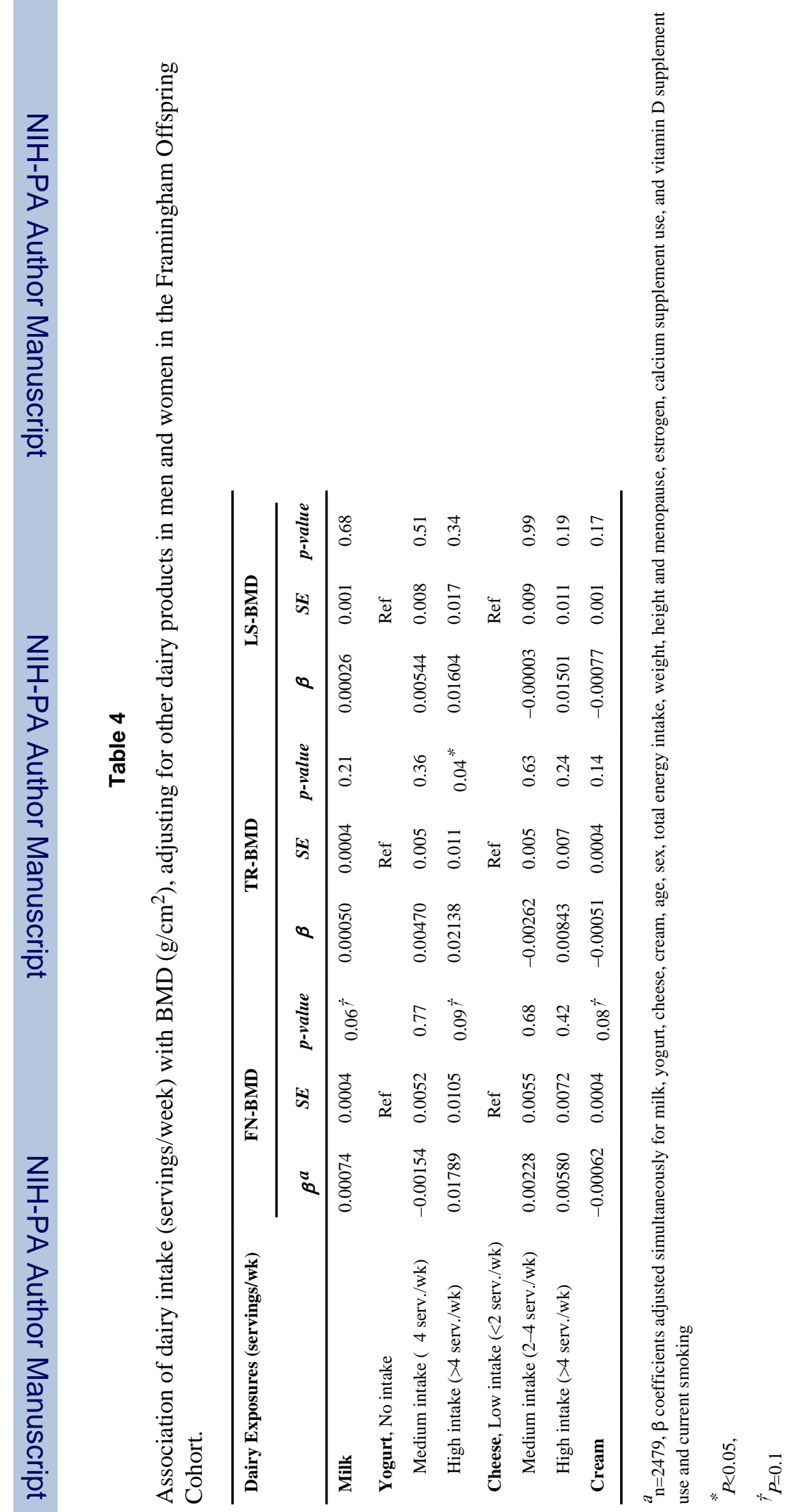

